PROCEEDINGS OF THE

AMERICAN MATHEMATICAL SOCIETY

Volume 137, Number 1, January 2009, Pages 189-193

S 0002-9939(08)09571-3

Article electronically published on August 1, 2008

\title{
ON A PRODUCT OF MODIFIED BESSEL FUNCTIONS
}

\author{
ÁRPÁD BARICZ \\ (Communicated by Carmen C. Chicone) \\ Dedicated to my son Koppány
}

\begin{abstract}
Let $I_{\nu}$ and $K_{\nu}$ denote the modified Bessel functions of the first and second kinds of order $\nu$. In this note we prove that the monotonicity of $u \mapsto$ $I_{\nu}(u) K_{\nu}(u)$ on $(0, \infty)$ for all $\nu \geq-1 / 2$ is an almost immediate consequence of the corresponding Turán type inequalities for the modified Bessel functions of the first and second kinds of order $\nu$. Moreover, we show that the function $u \mapsto$ $I_{\nu}(u) K_{\nu}(u)$ is strictly completely monotonic on $(0, \infty)$ for all $\nu \in[-1 / 2,1 / 2]$. At the end of this note, a conjecture is stated.
\end{abstract}

\section{Preliminaries And main Results}

Let $I_{\nu}$ and $K_{\nu}$ denote, as usual, the modified Bessel functions of the first and second kinds of order $\nu$. Recently, motivated by a problem which arises in biophysics, Penfold et al. [13, Theorem 3.1] proved, in a complicated way, that the product of the modified Bessel functions of the first and second kinds, i.e. $u \mapsto P_{\nu}(u)=$ $I_{\nu}(u) K_{\nu}(u)$, is strictly decreasing on $(0, \infty)$ for all $\nu \geq 0$. It is worth mentioning that this result for $\nu=n \geq 1$, a positive integer, was verified in 1950 by Phillips and Malin [14, Corollary 2.2]. In this note our aim is to show that using the idea of Phillips and Malin, the monotonicity of $u \mapsto P_{\nu}(u)$ for $\nu \geq-1 / 2$ can be verified easily by using the corresponding Turán type inequalities for modified Bessel functions. Moreover, we show that the function $u \mapsto I_{\nu}(u) K_{\nu}(u)$ is strictly completely monotonic on $(0, \infty)$ for all $\nu \in[-1 / 2,1 / 2]$, i.e. for all $u>0, \nu \in$ $[-1 / 2,1 / 2]$ and $m=0,1,2, \ldots$, we have

$$
(-1)^{m}\left[I_{\nu}(u) K_{\nu}(u)\right]^{(m)}>0 .
$$

In order to achieve our goal we improve some of the results of Phillips and Malin [14, Eq. 1] concerning bounds for the logarithmic derivatives of the modified Bessel and Hankel functions.

Our main result reads as follows:

Theorem 1. The following assertions are true:

a. the function $u \mapsto P_{\nu}(u)$ is strictly decreasing on $(0, \infty)$ for all $\nu \geq-1 / 2$;

Received by the editors December 13, 2007.

2000 Mathematics Subject Classification. Primary 33C10, 33C15.

Key words and phrases. Modified Bessel functions, complete monotonicity, Turán type inequalities.

This research was partially supported by the Institute of Mathematics, University of Debrecen, Hungary.

(C)2008 American Mathematical Society 
b. the function $u \mapsto P_{\nu}(u)$ is strictly completely monotonic on $(0, \infty)$ for all $\nu \in[-1 / 2,1 / 2]$

c. the function $u \mapsto u^{1 / 2-\nu} P_{\nu}(u)$ is strictly completely monotonic on $(0, \infty)$ for all $\nu \geq 1 / 2$.

\section{Proof of the MAIN RESUlt}

In this section our aim is to prove the assertions stated in Theorem 1 for the sake of completeness we also point out some historical facts concerning the corresponding Turán type inequalities.

a. Our strategy is as in the paper [14. Specifically, we first show that for all $u>0$ the following inequalities hold true:

$$
\begin{aligned}
& u I_{\nu}^{\prime}(u) / I_{\nu}(u)<\sqrt{u^{2}+\nu^{2}}, \quad \text { where } \nu \geq-1 / 2, \\
& u K_{\nu}^{\prime}(u) / K_{\nu}(u)<-\sqrt{u^{2}+\nu^{2}}, \quad \text { where } \nu \in \mathbb{R} .
\end{aligned}
$$

Inequality (2.1) for $\nu>0$ was first proved by Gronwall [6, Eq. 16] in 1932, motivated by a problem in wave mechanics; in 1950 it appeared also in Phillips and Malin's paper [14, Eq. 1] for $\nu=n \geq 1$, a positive integer. An equivalent form of (2.1) is known in the literature as Amos' inequality. More precisely, observe that in view of the recurrence relations

$$
I_{\nu-1}(u)=(\nu / u) I_{\nu}(u)+I_{\nu}^{\prime}(u) \text { and } I_{\nu+1}(u)=I_{\nu}^{\prime}(u)-(\nu / u) I_{\nu}(u),
$$

the Turán type inequality

$$
I_{\nu-1}(u) I_{\nu+1}(u)-\left[I_{\nu}(u)\right]^{2}<0
$$

is in fact equivalent to (2.1). Here we have used the known fact that the function $u \mapsto I_{\nu}(u)$ is increasing on $(0, \infty)$ for all $\nu>-1$. The Turán type inequality (2.3) for $\nu \geq 0$ was proved first in 1951 by Thiruvenkatachar and Nanjundiah [15], later in 1974 by Amos [1, p. 243], and in 1991 by Joshi and Bissu [8, p. 339]. It is worth mentioning that in fact the function $\nu \mapsto I_{\nu}(u)$ is log-concave on $(-1, \infty)$ for each fixed $u>0$ (see [5, Lemma 1.4] and [10, Theorem 3]). For more details on Turán type inequalities the interested reader is referred to the papers [2, 3], 44, [5, 8], [9], 10], 15] and to the references therein. Finally, let us note that in 1994 Lorch [10, p. 79] proved that (2.3) in fact holds for all $\nu \geq-1 / 2$ and $u>0$. From this we conclude that (2.1) holds too for all $\nu \geq-1 / 2$ and $u>0$.

Now, let us focus on the inequality (2.2). This inequality was proved for positive integers $\nu=n \geq 1$ by Phillips and Malin [14, Eq. 1] in 1950. For $\nu \in \mathbb{R}$ and $u>0$ consider the Turán type inequality

$$
K_{\nu-1}(u) K_{\nu+1}(u)-\left[K_{\nu}(u)\right]^{2}>0,
$$

which was proved in 1978 by Ismail and Muldoon [7, Lemma 2.2] and recently by the author [5, Theorem 1.13] using a completely different argument. Note that for $\nu>1 / 2$ the Turán type inequality (2.4) appears also on Laforgia and Natalini's paper [9, Eq. 2.18]. Observe that using the recurrence relations

$$
K_{\nu-1}(u)=-(\nu / u) K_{\nu}(u)-K_{\nu}^{\prime}(u) \text { and } K_{\nu+1}(u)=-K_{\nu}^{\prime}(u)+(\nu / u) K_{\nu}(u),
$$

we obtain that the Turán type inequality (2.4) is equivalent to the inequality

$$
\left[u K_{\nu}^{\prime}(u) / K_{\nu}(u)-\sqrt{u^{2}+\nu^{2}}\right]\left[u K_{\nu}^{\prime}(u) / K_{\nu}(u)+\sqrt{u^{2}+\nu^{2}}\right]>0
$$


which holds for all $\nu \in \mathbb{R}$ and $u>0$. Now, since the function $u \mapsto K_{\nu}(u)$ is decreasing on $(0, \infty)$ for all $\nu \in \mathbb{R}$, it follows that (2.4) is equivalent to (2.2).

Finally, by using the inequalities (2.1) and (2.2), we conclude that

$$
u\left[\log \left(P_{\nu}(u)\right)\right]^{\prime}=u\left[\log \left(I_{\nu}(u) K_{\nu}(u)\right)\right]^{\prime}<0 ;
$$

i.e. the function $u \mapsto P_{\nu}(u)$ is strictly decreasing on $(0, \infty)$ for all $\nu \geq-1 / 2$, as required.

b. Recall that in 1978 Nåsell [12, p. 2] proved that for all $u>0, \nu>-1 / 2$ and $m=0,1,2, \ldots$ the inequality

$$
(-1)^{m}\left[u^{-\nu} I_{\nu}(u) e^{-u}\right]^{(m)}>0
$$

holds true; i.e. the function $u \mapsto u^{-\nu} I_{\nu}(u) e^{-u}$ is strictly completely monotonic on $(0, \infty)$ for each $\nu>-1 / 2$. Since $I_{-1 / 2}(u)=\sqrt{2 / \pi} \cdot u^{-1 / 2} \cosh u$, it is easy to verify that for all $m=0,1,2, \ldots$ and $u>0$ we have

$$
(-1)^{m}\left[e^{-u} \cosh u\right]^{(m)}=\frac{1}{2}(-1)^{m}\left[e^{-2 u}+1\right]^{(m)}=2^{m-1} e^{-2 u}>0 .
$$

Thus, the function $u \mapsto u^{-\nu} I_{\nu}(u) e^{-u}$ is in fact strictly completely monotonic on $(0, \infty)$ for each $\nu \geq-1 / 2$. On the other hand, due to Miller and Samko 11, Theorem $5]$ it is known that the function $u \mapsto u^{\min \{\nu, 1 / 2\}} e^{u} K_{\nu}(u)$ is completely monotonic on $(0, \infty)$ for each $\nu \geq 0$. Clearly, from this it follows that the function $u \mapsto$ $u^{\nu} e^{u} K_{\nu}(u)$ is completely monotonic on $(0, \infty)$ for each $\nu \in[0,1 / 2]$. Now, since by the Leibniz formula for derivatives the product of a strictly completely monotonic and a completely monotonic function is strictly completely monotonic, we conclude that the function $u \mapsto P_{\nu}(u)$ is strictly completely monotonic on $(0, \infty)$ for all $\nu \in[0,1 / 2]$. In [13] Penfold et al. used a similar argument to that presented above in order to study the monotonicity of the function $u \mapsto P_{\nu}(u)$ for $\nu \in[0,1 / 2]$. However, it seems that the authors of [13] overlooked the fact that inequality (2.5) is strict. Now, observe that since $K_{\nu}(u)=K_{-\nu}(u)$, we obtain from Miller and Samko's result that the function $u \mapsto u^{-\nu} e^{u} K_{\nu}(u)$ is completely monotonic on $(0, \infty)$ for each $\nu \in[-1 / 2,0]$. Combining this with Nåsell's result, it follows that the function $u \mapsto u^{-2 \nu} P_{\nu}(u)$ is strictly completely monotonic on $(0, \infty)$ for each $\nu \in[-1 / 2,0]$. Finally, since $u \mapsto u^{2 \nu}$ is strictly completely monotonic on $(0, \infty)$ for each $\nu \in[-1 / 2,0]$, we conclude that the function $u \mapsto P_{\nu}(u)$ is strictly completely monotonic on $(0, \infty)$ for each $\nu \in[-1 / 2,0]$, and hence for each $\nu \in[-1 / 2,1 / 2]$.

c. Since the function $u \mapsto u^{-\nu} I_{\nu}(u) e^{-u}$ is strictly completely monotonic on $(0, \infty)$ for each $\nu \geq-1 / 2$ and the function $u \mapsto u^{1 / 2} e^{u} K_{\nu}(u)$ is completely monotonic on $(0, \infty)$ for each $\nu \geq 1 / 2$, the result follows.

\section{Concluding Remarks And Further Results}

In this note we have shown that the monotonicity of the function $u \mapsto I_{\nu}(u) K_{\nu}(u)$ can be deduced easily by using the Turán type inequalities (2.3) and (2.4). In the process of deducing this result we have improved the range of validity for the inequalities (2.1) and (2.2), which are in fact equivalent to the inequalities (2.3) and (2.4). We note that our approach is much simpler than the methods used in 13 and [14.

Motivated by the inequalities (2.1) and (2.2), in what follows we are mainly interested in extensions of inequalities (2.1) and (2.2). Phillips and Malin 14, 
Eq. 1] proved that for each $u>0$ and positive integer $\nu$ the inequality

$$
u I_{\nu}^{\prime}(u) / I_{\nu}(u)>\sqrt{u^{2} \nu /(\nu+1)+\nu^{2}}
$$

holds true. We note that in fact (3.1) holds true for all $\nu>0$. To prove this consider the Turán type inequality

$$
(\nu+1) I_{\nu-1}(u) I_{\nu+1}(u)-\nu\left[I_{\nu}(u)\right]^{2}>0,
$$

which was proved, using completely different arguments, in 1951 by Thiruvenkatachar and Nanjundiah [15, in 1991 by Joshi and Bissu [8, p. 339], and recently by the author [4, Theorem 1]. Using again the corresponding recurrence relations, as in the proof of (2.1), it is easy to see that (3.1) is equivalent to (3.2); thus the result of Phillips and Malin holds in fact for all $\nu>0$.

In the same paper Phillips and Malin [14, Eq. 1] showed that for each $u>0$ and positive integer $\nu$ the inequality

$$
u K_{\nu}^{\prime}(u) / K_{\nu}(u)>-\sqrt{u^{2} \nu /(\nu-1)+\nu^{2}}
$$

holds true. From this we obtain that for each positive integer $\nu$ and $u>0$,

$$
\left[u K_{\nu}^{\prime}(u) / K_{\nu}(u)+\sqrt{u^{2} \nu /(\nu-1)+\nu^{2}}\right]\left[u K_{\nu}^{\prime}(u) / K_{\nu}(u)-\sqrt{u^{2} \nu /(\nu-1)+\nu^{2}}\right]<0 .
$$

Then, using the corresponding recurrence relations, as in the proof of (2.2), we obtain that the Turán type inequality

$$
(\nu-1) K_{\nu-1}(u) K_{\nu+1}(u)-(2 \nu-1)\left[K_{\nu}(u)\right]^{2}<0
$$

holds true for all positive integers $\nu$ and all $u>0$. Moreover, using again the recurrence relation $K_{\nu}(u)=K_{-\nu}(u)$, it follows from (3.3) that

$$
(\nu+1) K_{\nu-1}(u) K_{\nu+1}(u)-(2 \nu+1)\left[K_{\nu}(u)\right]^{2}>0
$$

holds true for all negative integers $\nu$ and all $u>0$. As far as we know these Turán type inequalities are new, and based on numerical experiments we conjecture the following:

Conjecture. The inequality (3.3) holds for all real $\nu \geq 0$, while (3.4) holds for all real $\nu \leq 0$.

\section{REFERENCES}

[1] Amos, D.E., 1974, Computation of modified Bessel functions and their ratios. Mathematics of Computation, 28, 239-251. MR0333287(48:11612)

[2] Baricz, Á., 2007, Turán type inequalities for generalized complete elliptic integrals. Mathematische Zeithschrift, 256(4), 895-911. MR22308896

[3] Baricz, Á., 2008, Turán type inequalities for hypergeometric functions. Proceedings of the American Mathematical Society, 136(9), 3223-3229.

[4] Baricz, Á., 2008, Functional inequalities involving Bessel and modified Bessel functions of the first kind. Expositiones Mathematicae, 26(3), 279-293.

[5] Baricz, Á., Turán type inequalities for some probability density functions. Studia Scientiarium Mathematicarum Hungarica (submitted).

[6] Gronwall, T.H., 1932, An inequality for the Bessel functions of the first kind with imaginary argument. Annals of Mathematics, 33(2), 275-278. MR1503051

[7] Ismail, M.E.H. and Muldoon, M.E., 1978, Monotonicity of the zeros of a cross-product of Bessel functions. SIAM Journal on Mathematical Analysis, 9(4), 759-767. MR0486686 $(58: 6388)$ 
[8] Joshi, C.M. and Bissu, S.K., 1991, Some inequalities of Bessel and modified Bessel functions. Journal of the Australian Mathematical Society (Series A), 50, 333-342. MR 1094928 (92d:33007)

[9] Laforgia, A. and Natalini, P., 2006, On some Turán-type inequalities. Journal of Inequalities and Applications, 2006, Article 29828, 6 pp. MR2221215 (2007c:26028)

[10] Lorch, L., 1994, Monotonicity of the zeros of a cross product of Bessel functions. Methods and Applications of Analysis, 1(1), 75-80. MR1260384 (95b:33008)

[11] Miller, K.S. and Samko, G., 2001, Completely monotonic functions. Integral Transforms and Special Functions, 12, 389-402. MR:1872377 (2002j:26006)

[12] Nåsell, I., 1978, Rational bounds for ratios of modified Bessel functions. SIAM Journal on Mathematical Analysis, 9, 1-11. MR0466662 (57:6539)

[13] Penfold, R., Vanden-Broeck, J.-M. and Grandison, S., 2007, Monotonicity of some modified Bessel function products. Integral Transforms and Special Functions, 18(2), 139-144. MR2290352 (2007m:33015)

[14] Phillips, R.S. and Malin, H., 1950, Bessel function approximations. American Journal of Mathematics, 72, 407-418. MR0035346 (11:720d)

[15] Thiruvenkatachar, V.R. and Nanjundiah, T.S., 1951, Inequalities concerning Bessel functions and orthogonal polynomials. Proceedings of the Indian Academy of Sciences. Section A, 33, 373-384. MR0048635(14:44b)

Faculty of Economics, Babeş-Bolyai University, RO-400591 Cluj-Napoca, Romania

E-mail address: bariczocsi@yahoo.com 